\title{
Self-assembling doxorubicin silk hydrogels for the focal treatment of primary breast cancer
}

\author{
F. Philipp Seib [Dr.], Eleanor M. Pritchard [Dr.], and David L. Kaplan [Prof.] \\ Tufts University, Department of Biomedical Engineering, 4 Colby Street, Medford, MA 02155, \\ USA
}

David L. Kaplan: David.Kaplan@tufts.edu

\begin{abstract}
Standard care for early stage breast cancer includes tumor resection and local radiotherapy to achieve long-term remission. Systemic chemotherapy provides only low locoregional control of the disease; therefore, we describe self-assembling silk hydrogels that can retain and then deliver doxorubicin locally. Self-assembling silk hydrogels show no swelling, are readily loaded with doxorubicin under aqueous conditions and release drug over 4 weeks in amounts that can be finetuned by varying the silk content. Following successful in vitro studies, locally injected silk hydrogels loaded with doxorubicin show excellent antitumor response in mice, outperforming the equivalent amount of doxorubicin delivered intravenously. In addition to reducing primary tumor growth, doxorubicin-loaded silk hydrogels reduce metastatic spread and are well tolerated in vivo. Thus, silk hydrogels are well suited for the local delivery of chemotherapy and provide a promising approach to improve locoregional control of breast cancer.
\end{abstract}

\section{Keywords}

Silk hydrogel; adriamycin; drug delivery; Cancer

\section{Introduction}

Each year in the United States alone, more than 230,000 new breast cancer cases are diagnosed and 40,000 women die as the result of the disease ${ }^{[1]}$. For early stage breast cancer, surgical resection of the tumor followed by localized radiotherapy to the affected breast is standard clinical practice ${ }^{22]}$; this treatment strategy is supported by long-term follow up studies (e.g.. ${ }^{[3,4]}$ ). Clinical trials have demonstrated that high-risk, premenopausal women benefit from local radiotherapy, which reduces ipsilateral tumor recurrence and enhances overall survival ${ }^{[5]}$. Therefore, locoregional control is necessary to improve long-term clinical outcomes. Taking into account that systemic chemotherapy provides limited control over the locoregional disease ${ }^{[2]}$, direct application of chemotherapy to the primary tumor site might improve clinical outcomes. However, no studies have been reported that specifically set out to design focal delivery systems for breast cancer applications.

Localized chemotherapy is routinely used for a number of malignancies. For example, bladder cancer is often treated with intravesical chemotherapy and high-grade malignant glioma is treated by placing carmustine-containing synthetic copolymer wafers (Gliadel ${ }^{\circledR}$

Correspondence to: David L. Kaplan, David. Kaplan@tufts . edu.

Supporting Information is available online from Wiley InterScience or from the author. 
Wafer) at the site of tumor resection ${ }^{[6,7]}$. A number of polyethylene glycol (PEG) hydrogelbased systems that are tailored to respond to a stimulus such as temperature, ionic strength or $\mathrm{pH}^{\left[{ }^{8-12]}\right.}$ to trigger controlled drug release are in preclinical development. Other hydrogel systems include poly(organophosphazane) ${ }^{[8]}$ polymers, PLGA-PEG-PLGA triblock compolymers ${ }^{[10]}$ or poly(ester-carbonate)-composites ${ }^{[13]}$. A major limitation for PEGbased systems is their resistance to biodegradation. Therefore, such delivery platforms need to be carefully designed to avoid the surgical removal of the device. One strategy includes the use of proteinase-sensitive sequences in the polymeric backbone, which was first used in $N$-(2-hydroxypropyl)methacrylamide gels ${ }^{[14]}$. Synthetic hydrogel systems require chemical crosslinking that necessitates the use of potentially harmful agents such as organic solvents, chemical initiators or UV irradiation. Residual chemicals can leach from hydrogels, and UV-based polymerization techniques are incompatible with many anticancer drugs (e.g., doxorubicin, daunorubicin, cyclophosphamide). Synthetic hydrogels are therefore often plagued by poor biocompatibility and are challenging from a drug formulation perspective. To date, no hydrogel system has been introduced into the clinic that can overcome all of these limitations.

In addition to the paucity of synthetic hydrogels for breast cancer therapy, there is also a lack of naturally derived biopolymers suitable for this application. A notable exception is silk. Silk has a robust safety record in humans ${ }^{[15]}$ and is known to degrade in vivo ${ }^{[16]}$. The degradation profile of silk can be fine-tuned to range from hours (low crystalline silk) to 12 months or more (high crystalline silk) ${ }^{[16]}$. Overall, silk drug carriers, such as microparticles and films, are able to stabilize the payload and control drug release using endogenous parameters such as the crystallinity and molecular weight of the silk fibroin ${ }^{[17,18]}$. The aforementioned studies have focused on demonstrating the potential of silk to serve as a delivery system per se; this effort also included the development of mathematical models that predicted the release of model drugs (e.g.. ${ }^{[19]}$ ). Although a number of silk formulations have been reported, there have been no studies that examined the therapeutic potential of silk hydrogels for cancer therapy either in vitro or in vivo. We therefore developed selfassembling silk hydrogels to deliver doxorubicin because this anticancer agent is commonly used in breast cancer chemotherapy ${ }^{[2,20]}$ and would benefit significantly from an improved safety profile ${ }^{[21]}$. Next, we tested silk hydrogels loaded with doxorubicin for focal breast cancer delivery and compared this delivery system to systemic doxorubicin. The resulting anticancer silk hydrogels improved both the safety and efficacy of doxorubicin in human breast cancer xenografts. Self-assembling silk hydrogels allowed drug loading under ambient conditions and led to sustained doxorubicin delivery, suggesting that this approach is a promising avenue for focal breast cancer therapy.

\section{Results}

\subsection{Manufacture of self-assembling doxorubicin-loaded silk hydrogels}

The impact of selected processing parameters on gel formation was determined by monitoring gelation-induced light scattering (Figure 1a). Sonication time had a major impact on gelation kinetics; following a 45 second sonication time, hydrogel formation was completed within 60 minutes, whereas sonicating silk for 30 seconds increased the gelation time to 300 minutes at room temperature. The optical properties of the hydrogels depended on the initial amount of silk in solution. Hydrogels with a high silk content caused more light scattering than those with a low silk content (Figure 1a). Next, the swelling behavior of silk hydrogels was tested. No swelling was observed for either $2 \mathrm{wt} \%$ or $4 \mathrm{wt} \%$ hydrogels after $24 \mathrm{~h}$ (Figure 1b). Silk hydrogels were also subjected to in vitro stability studies (Figure 1c). In PBS, due to the absence of proteolytic enzymes, hydrogels showed no change in mass, whereas in a-chymotrypsin, they showed significant degradation that was greatest for the $2 \mathrm{wt} \%$ hydrogels (Figure 1c). To determine the ability of silk hydrogels to control 
doxorubicin release, both the amount of silk and the degumming times were systematically studied. For silk hydrogels degummed for $20 \mathrm{~min}$ or $45 \mathrm{~min}$, there were no significant differences in drug release over 4 weeks (Figure 1d). However, the weight percentage of silk in the hydrogel had a significant impact on drug release. Drug release was the slowest for 6 wt $\%$ silk hydrogels, resulting in a cumulative doxorubicin amount of $17 \%$ at 4 weeks. The cumulative amount released from $4 \mathrm{wt} \%$ and $2 \mathrm{wt} \%$ gels were $22 \%$ and $27 \%$, respectively (Figure 1d). For all preparations, the doxorubicin release was rapid over the first 10 days (65\% of total cumulative release) and declined over the remaining time course of the study. To permit robust self-assembly of silk hydrogels, a minimum amount of $1.0 \mathrm{wt} \%$ silk was necessary; in line with long term doxorubicin release studies, gels with the lowest amount of silk released $30 \%$ of the loaded drug over the first week with subsequent less for $1.5 \mathrm{wt} \%$ > $2.0 \mathrm{wt} \%>4.0 \mathrm{wt} \%>6.0 \mathrm{wt} \%$ gels (Figure S2). In addition to the amount of silk, hydrogels could be readily tailored to contain different amounts of doxorubicin (Figure 1e and Figure S1). For in vivo studies, pre-filled syringes that contained $2 \mathrm{wt} \%$ hydrogels and $40 \mu \mathrm{g}$ of doxorubicin were used (Figure 1f). These hydrogel preparations were selected for the remaining studies because they could be readily injected both in vitro (Figure 1f) and in vivo.

\subsection{In vitro cytotoxicity of doxorubicin-loaded silk hydrogels}

First, the cytotoxicity of hydrogels loaded with doxorubicin and free drug was evaluated using MDA-MB-231 and MCF-7 (human) breast cancer cell lines and 3-day exposures (Figure 2a, b, Figure S2). In this short-term cytotoxicity assay, both $2 \mathrm{wt} \%$ and $4 \mathrm{wt} \%$ silk hydrogels loaded with $40 \mu \mathrm{g}$ of doxorubicin were compared to free drug. The amount of doxorubicin released over this 3-day culture period from silk hydrogels was $1.2 \mu \mathrm{g}$ and 2.4 $\mu \mathrm{g}$ for $4 \mathrm{wt} \%$ and $2 \mathrm{wt} \%$ hydrogels, respectively. Therefore, the control cultures used $1.2 \mu \mathrm{g}$ and $2.4 \mu \mathrm{g}$ of free doxorubicin. MCF-7 viability was reduced to $10 \%$ following exposure to free doxorubicin and to $20 \%$ for silk hydrogels (Figure 2a). At the $1.2 \mu \mathrm{g}$ and $2.4 \mu \mathrm{g}$ doxorubicin concentration cell viability was similar (Figure S3). This non-linear biological response is often observed in vitro with cytotoxic agents like doxorubicin; in part, cell cycle, exposure time and endogenous resistance mechanisms are thought to contribute to this observation ${ }^{[20]}$. For MDA-MB-231 cells, viability was reduced to $23 \%$ for all treatment groups (Figure $2 \mathrm{~b}$ ). Cultures treated with silk hydrogels without drug showed no significant change in cell viability when compared to control cultures that had not been treated. In addition to the 3-day cytotoxicity assay, a 12-day longer-term assay was performed (Figure 2c). In an attempt to mimic the in vivo pharmacokinetics of intravenous doxorubicin, culture medium was replaced throughout the 12-day culture period. Furthermore, cultures were reseeded at day 6 to mimic disease relapse. Control cultures of MDA-MB-231 and MCF-7 demonstrated exponential cell growth during days 2 through 6 and less growth during the remaining days in culture. Doxorubicin-treated groups showed no or minimal growth during the first 6 days in culture. However, cells that had been treated with free doxorubicin showed significant outgrowth over the remaining course of the study. In contrast, silk hydrogels loaded with doxorubicin were able to inhibit cell growth throughout the study (12 days). The ability of doxorubicin-loaded silk films to inhibit cell growth for extended periods of time in vitro warranted in vivo studies.

\subsection{In vivo antitumor studies of doxorubicin-loaded silk hydrogels}

The therapeutic impact of delivering the equivalent amount of doxorubicin by silk hydrogels or by a bolus intravenous injection was studied (Figure 3). Orthotopic tumors were induced, and therapeutic intervention was initiated once tumors were established (Figure S4). From week 3 onward, doxorubicin-loaded silk hydrogels showed better antitumor activity than the equivalent amount of doxorubicin delivered intravenously (Figure 3a). At week 6, significant tumor response was seen for doxorubicin-loaded hydrogels but not for 
intravenous doxorubicin (Figure 3b, c). The animals were euthanized at week 6 (Figure 3c), and the primary tumors were removed and weighed (Figure $3 \mathrm{~d}$ ). The reduction in tumor burden was greater for mice that had received doxorubicin-loaded silk hydrogels than for mice that had been treated with intravenous doxorubicin (Figure 3d). There was a good correlation between the tumor weights and the cancer cell-associated bioluminescence signals detected by non-invasive imaging (Figure 3e). Two of the five animals treated with silk hydrogels loaded with doxorubicin showed complete tumor regression as determined by bioluminescence imaging and necropsy. In addition to primary tumor growth, metastatic spread into bones, lung, liver and brain was determined (Figure 3f). All untreated control mice had bone metastases and a high frequency of lung and liver metastases $(60 \%)$ followed by metastatic spread to the brain $(40 \%)$. However, animals that received intravenous doxorubicin had a reduced frequency of bone (80\%) and liver (40\%) metastases and no brain metastases. The clinical outcome was improved for animals that received silk hydrogels containing doxorubicin; there was no lung metastasis and a low frequency of brain metastases (20\%). The frequency of liver metastases was comparable to that seen for mice treated with intravenous doxorubicin (Figure 3f).

\subsection{Autopsy of mice treated with self-assembling silk hydrogels}

Autopsy indicated that the silk hydrogels were surrounded by a fibrous capsule (Figure S5a). Further histological examination of hematoxylin and eosin-stained sections demonstrated fibroplastic growth and collagen deposition at the periphery of the silk hydrogel, with few or no cells in the inner mass of the gel (Figure S5b). It was noticed that the thickness of the fibrous capsule varied from $20 \mu \mathrm{m}$ to $300 \mu \mathrm{m}$. There were no signs of acute or chronic inflammation. The heart weight was used to examine systemic doxorubicin-associated toxicity [22]. For animals that were treated with doxorubicin, there was significant reduction in heart weight, which was more pronounced following intravenous dosing than following treatment with hydrogels (Figure 4a). To examine cardiotoxicity further, hearts were stained with hematoxylin and eosin (H\&E) and examined (Figure 4b). All treatment groups showed similar cellular morphology and H\&E staining. There were no signs of myocardial remodeling or scarring in any of the animals that had received either intravenous doxorubicin or doxorubicin-loaded silk gels. The local tissue response to doxorubicin-loaded silk hydrogels was examined at the end of the study. There were no macroscopic changes in any of the treatment groups (Figure S6). Histology of the samples showed that animals treated with doxorubicin silk hydrogels had a mild tissue response characterized by cellular infiltration and matrix deposition (Figure S6).

\section{Discussion}

The majority of breast-cancer-related deaths are from blood-born metastases ${ }^{[23]}$. Therefore, the use of localized chemotherapy to treat the disease might appear counterintuitive. However, emerging evidence from human clinical trials suggests ${ }^{[5]}$ that good locoregional control of the disease is essential to reduce the risk of ipsilateral and distant tumor recurrence ${ }^{[24]}$. Although systemic chemotherapy is routinely used in early stage breast cancer patients to target disseminated breast cancer cells, such treatment schedules provide only poor control over the locoregional disease ${ }^{[2]}$.

We therefore set out to develop a local delivery system that is injectable, biodegradable and capable of delivering a clinically relevant chemotherapeutic agent ${ }^{[20]}$. We demonstrate that self-assembling ilk-based hydrogels are readily manufactured and loaded with the drug doxorubicin, do not exhibit swelling and are degradable. Following sonication, these gels formed spontaneously because of the physical crosslinking of hydrophobic silk repeats. Overall, this results in a physically crosslinked network that is stabilized by $\beta$-sheets [25] (Figure S2c). To achieve a stable network, a minimum of silk fibroin ( 1 wt $\%)$ is required 
for the self-assembly process. By adjusting the amount of silk, it was possible to tailor drug release; by reducing the amount of silk to $1 \mathrm{wt} \%$, drug release could be increased 4.5 -fold when compared to $6 \mathrm{wt} \%$ gels. The presence of the hydrophobic core repeats of silk likely facilitated doxorubicin adsorption ${ }^{[15]}$ because preliminary adsorption studies with silk films have indicated that binding affinity is often dependent on crystallinity. Therefore, the greater the amount of $\beta$-sheet in the gels, the stronger the adsorptive capacity of doxorubicin to the silk network resulting in an overall slower drug release (Figure S2c). In vitro studies demonstrated that drug release was dependent on the adsorption affinity of doxorubicin to silk, because we observed no gel degradation that could account for drug release. However, silk hydrogels are (bio)degradable following exposure to proteases like a-chymotrpysin.

We tested silk hydrogels for their ability to release doxorubicin, which led to a significant reduction in the cell viability of human breast cancer cell lines in short-term in vitro cultures. For these studies, we used $2 \mathrm{wt} \%$ and $4 \mathrm{wt} \%$ hydrogels as these formulations could be easily injected, retained their physical properties and provided the desired drug release kinetics for in vitro studies. By mimicking disease relapse in long-term studies, we have also shown that silk hydrogels loaded with doxorubicin minimized cell growth throughout the 12-day culture period. The ability of silk hydrogels to provide superior disease control was apparent because only samples that were treated with freely diffusible doxorubicin allowed outgrowth of breast cancer cells. We wished to see whether our in vitro observations would translate into cancer therapy in vivo and therefore used a humanized orthotopic breast cancer model. Although systemic treatment of mice with doxorubicin reduced the tumor burden and metastatic spread, a better response was observed for doxorubicin-loaded silk hydrogels. In addition to reduced tumor growth, we observed a complete response in 2 of 5 mice and an overall reduced metastatic spread in all animals. It appears that the good locoregional control achieved in this study mimicked some of the features seen in human clinical trials ${ }^{[5]}$, including reduced metastasis.

Although myelosuppression is a serious side effect associated with chemotherapy regimens containing doxorubicin, a real clinical challenge is the cardiotoxicity associated with doxorubicin ${ }^{[21]}$. Acute left ventricular failure limits the total cumulative doxorubicin dose in patients to $300 \mathrm{mg} / \mathrm{m}^{2}$ [21]. Previous studies have used heart weight as a readout of doxorubicin-associated cardiotoxicity ${ }^{[22]}$. In the present study, the greatest reduction in heart weight was seen following intravenous doxorubicin administration. Therefore, targeting the drug to the tumor site resulted in a significant reduction of systemic side effects. Histological examination of hearts did not show doxorubicin-associated changes of the myocardium as reported previously $[22,26,27]$. The $2-4$ times lower cumulative doxorubicin dose of the present study is likely to contribute to improved myocardial health.

The biocompatibility and biodegradation of silk hydrogels makes them a useful biomaterial delivery platform for the development of localized chemotherapy. The focus of this study was their application in breast cancer, though we expect that this platform technology could be easily translated to a number of other cancers where local minimally invasive treatment is desirable. In the present study, we used pre-gelled silk hydrogels that were injected in vivo. There are a number of advantages of such a system that include but are not limited to (1) consistent preparations with minimal batch to batch variation, (2) easy storage and (3) easy handling during the procedure. However, we are aware that these pre-formed gels do not conform to the entire surface of the tumor. Therefore, in situ hydrogel formation would be useful to overcome this limitation without compromising the key features of our hydrogel system. This approach is feasible based on our prior studies to show the range and versatility of control of such systems ${ }^{[28]}$. Although the applicability of silk hydrogels for human use remains to be established, the extensive experience with silk-based material in humans (e.g., load bearing applications) bodes well for the future of such an approach. 


\section{Conclusion}

In conclusion, adjusting the properties of silk hydrogels provided control over doxorubicin release that minimized systemic side effects but maximized therapeutic impact. This study demonstrated that silk biopolymers can serve as a valuable local delivery platform for breast cancer research and provides the foundation for the development of silk-based hydrogels for cancer research and therapy.

\section{Experimental Section}

\section{Doxorubicin-loaded silk hydrogels for in vitro and in vivo studies}

Bombyx mori fibroin solution was prepared as described previously [29]. Briefly, cocoons were cut into $\sim 25 \mathrm{~mm}^{2}$ pieces, boiled for either $20 \mathrm{~min}$ or $45 \mathrm{~min}$ in a $25 \mathrm{mM} \mathrm{Na}_{2} \mathrm{CO}_{3}$ solution and then rinsed in $\mathrm{ddH}_{2} \mathrm{O}$ to remove sericin proteins (i.e., degumming). Extracted silk fibroin was subsequently air dried and dissolved in $9.3 \mathrm{M} \mathrm{LiBr}$ solution at $60^{\circ} \mathrm{C}$, yielding a $20 \mathrm{wt} \%$ solution. This solution was dialyzed (molecular weight cutoff 3,500 ) against $\mathrm{ddH}_{2} \mathrm{O}$ for $48 \mathrm{~h}$ to remove the $\mathrm{LiBr}$ salt. The resulting aqueous silk fibroin solution was cleared by centrifugation. Silk hydrogel preparations [29] and their biophysical properties have been reported elsewhere [28]. Briefly, gels were prepared by degumming silk solutions of $2 \mathrm{wt} \%, 4 \mathrm{wt} \%$ or $6 \mathrm{wt} \%$ for either $20 \mathrm{~min}$ or $45 \mathrm{~min}$ and then sonicating them using a Branson Digital Sonifier 450 (Branson Ultrasonics, Danbury, CT, USA) at $15 \%$ amplitude for 30-90 seconds. Next, aqueous doxorubicin was mixed into the sonicated silk solution prior to the onset of gelation. The doxorubicin loadings ranged from 8 to 250 $\mu \mathrm{g} / \mathrm{gel}$. For in vitro studies, silk of either $2 \mathrm{wt} \%$ or $4 \mathrm{wt} \%$ was degummed for $45 \mathrm{~min}$, sterilized, filtered and subsequently processed to yield gels $\pm 40 \mu \mathrm{g}$ doxorubicin/gel. For in vivo studies, $2 \mathrm{wt} \%$ sterile silk was processed and loaded into syringes prior to gelation to yield ready-to-use syringes with $100 \mu \mathrm{l}$ of hydrogel and $40 \mu \mathrm{g}$ of doxorubicin. During hydrogel processing, 10x phosphate buffered saline (PBS) was used to obtain physiological osmolarity of the final preparation.

\section{Silk hydrogels: Swelling and release kinetics of doxorubicin}

To determine hydrogel swelling, the starting weight of freshly prepared gels was recorded. Next, samples were allowed to swell for $24 \mathrm{~h}$ in PBS and measured again, and the percentage weight gain was calculated. To monitor doxorubicin release from silk hydrogels, samples were incubated with $\mathrm{PBS}$ at $37^{\circ} \mathrm{C}$. Cumulative drug release was monitored by removing and replacing the buffer every $24 \mathrm{~h}$ and measuring doxorubicin-associated fluorescence (excitation wavelength $485 \mathrm{~nm}$, emission wavelength $590 \mathrm{~nm}$ ).

\section{In vitro degradation studies of silk hydrogels}

Silk hydrogels were either incubated with PBS or with a Tris-CaCl $\mathrm{Cuffer}_{2}(100 \mathrm{mM}$ Tris$\mathrm{HCl}, \mathrm{pH} 7.8,50 \mathrm{mM} \mathrm{CaCl}_{2}$ ) containing $2 \mathrm{U}$ of a-chymotrypsin per $400 \mathrm{mg}$ of silk hydrogel (Sigma-Aldrich, St Louis, IL, USA) to represent degradation of the silk by proteolytic enzymes in vivo (Arai et al., 2004). Solutions were replaced every 3 days, and at the indicated time, the remaining mass was determined by weighing.

\section{In vitro cytotoxicity studies}

The breast cancer cell lines MDA-MB-231 and MCF-7 were obtained from ATCC (Manassas, VA, USA). Cells were maintained in a humidified atmosphere of $5 \% \mathrm{CO}_{2}$ at $37^{\circ} \mathrm{C}$, and cultures were routinely subcultured every $2-3$ days. The MDA-MB-231 cells were grown in RPMI 1640 with $10 \%$ v/v FBS. The MCF-7 cells were cultured in DMEM ( $4.5 \mathrm{~g}$ glucose, $110 \mathrm{mg}$ sodium pyruvate) supplemented with $10 \% \mathrm{v} / \mathrm{v}$ FBS and $10 \mu \mathrm{g} / \mathrm{ml}$ insulin. The in vitro toxicity of doxorubicin-loaded silk hydrogels and free doxorubicin 
samples was determined by plating $2 \times 10^{4}$ cells $/ \mathrm{cm}^{2}$ in the lower chamber of Transwells. Cultures were allowed to recover for $24 \mathrm{~h}$. Next, silk hydrogels $(100 \mu \mathrm{l}) \pm 40 \mu \mathrm{g}$ doxorubicin loading were added to chamber inserts $(0.4 \mu \mathrm{m}$ pore size, Corning, New York USA). As a control, free doxorubicin at $40 \mu \mathrm{g}$ was added to control wells to represent the total drug loading. The amount of doxorubicin released over $72 \mathrm{~h}$ from $4 \mathrm{wt} \%$ and $2 \mathrm{wt} \%$ silk hydrogels was $1.2 \mu \mathrm{g}$ and $2.4 \mu \mathrm{g}$, respectively. Therefore, $1.2 \mu \mathrm{g}$ and $2.4 \mu \mathrm{g}$ of free doxorubicin were also added to the respective control wells. For endpoint studies, cell viability was determined after a $72 \mathrm{~h}$ exposure time with (3-(4,5-dimethylthiazol-2-yl)-2,5diphenyltetrazolium bromide (MTT) at $5 \mathrm{mg} / \mathrm{ml}$ as a substrate. Following a $5 \mathrm{~h}$ incubation period, formazan was solubilized with dimethylsulfoxide, and the absorbance was measured at $560 \mathrm{~nm}$. For long-term studies, analogous studies were performed but using AlamarBlue (Invitrogen, Grand Island, NY, USA) instead of MTT and an initial seeding density of $1 \times$ $10^{4}$ cells $/ \mathrm{cm}^{2}$. At the indicated times, $50 \mu \mathrm{l}$ of AlamarBlue was added to the culture medium, and cell viability was measured after a $4 \mathrm{~h}$ incubation period by monitoring fluorescence (excitation wavelength $550 \mathrm{~nm}$, emission wavelength $590 \mathrm{~nm}$ ) according to the manufacturer's protocol. Next, medium was replaced with fresh culture medium, and cultures were continued. To mimic disease relapse, culture inserts were re-seeded at day 6 with $1 \times 10^{4}$ cells $/ \mathrm{cm}^{2}$, and viability was monitored as detailed above. The only culture that was not re-seeded was the control group because it reached confluency at this time.

\section{In vivo tumor studies and necropsy}

All in vivo studies were approved by the Institutional Animal Care and Use Committee (Protocol B2010-101), and animals were maintained under the guidelines established by the National Institute of Health and Tufts University. Tumor xenografts were induced using MDA-MB-231-derived tumor cells that metastasized following orthotopic injection in mice and carried the firefly luciferase gene to permit in vivo bioluminescence imaging [30]. Female NOD/SCID mice (NOD.CB17-Prkdc ${ }^{\text {scid }} / \mathrm{NcrCrl}$ ) aged 6 to 10 weeks were obtained from Charles River (Wilmington, USA). For surgeries, animals were anesthetized using isoflurane and shaved, and the surgical area was cleaned. A total of $5 \times 10^{5}$ cells in $20 \mu \mathrm{l}$ of Matrigel (BD Biosciences, Bedford USA) were injected bilaterally into the $4^{\text {th }}$ or $5^{\text {th }}$ mammary fat pad using a Hamilton syringe equipped with a 22 Gauge needle. Following tumor induction, mice were treated at day 12 with $100 \mu \mathrm{l}$ of a $2 \mathrm{wt} \%$ silk hydrogel containing $40 \mu \mathrm{g}$ doxorubicin (denoted as silk hydrogel + doxorubicin). The doxorubicin dose and delivery system were optimized to maximize antitumor response. Mice received bilateral injections close to the tumor sites (i.e., $80 \mu \mathrm{g}$ of doxorubicin/mouse) (N.B. no intratumor injections). As a control, the equivalent doxorubicin dose of $80 \mu \mathrm{g}$ was administered in $100 \mu \mathrm{l}$ of PBS via tail vein injection to the respective mice (denoted as doxorubicin i.v.). For each treatment group, 5 animals were used. Disease progression was monitored weekly by tumor cell-associated bioluminescence imaging using the Xenogen IVIS 200 imaging system controlled by the Living Image Software 4.2 (Caliper Life Sciences, Hopkinton USA). At the endpoint of the study, brain, lung, liver and bones were examined for metastasis by dissecting them from the carcass. Tibias and femurs of hind legs were harvested and dissected free from muscle and tendon and used as the bone samples. Organs were imaged at maximum sensitivity to detect metastatic cancer cells. Tissues were scored for the presence or absence of metastasis. Primary tumors were dissected and weighed.

Histology samples were fixed in buffered formalin and processed for paraffin embedding. Samples were sectioned at the transverse plane at two levels and stained with H\&E. Hearts were also removed and weighed and subsequently processed for $\mathrm{H} \& \mathrm{E}$ staining as detailed before. A board-certified veterinarian pathologist examined the cardiac sections for signs of doxorubicin-associated toxicity using a scoring system described previously [31]. 


\section{Statistical analysis}

Data were analyzed using GraphPad Instat 3.06 (GraphPad Software, La Jolla USA). Two samples were analyzed using Student's t-test to determine statistical differences $(\mathrm{P} \leq 0.05)$. Multiple comparisons were evaluated by one-way analysis of variance (ANOVA) followed by Dunnett's post hoc tests to evaluate the statistical differences $(* \mathrm{P} \leq 0.05, * * \mathrm{P} \leq 0.001$, $* * * \mathrm{P} \leq 0.0001$ ) among samples and controls. Where indicated, Bonferroni's post hoc test was used. All error bars indicate the standard deviation (SD) except for the in vivo study for which the error bars indicate the standard of the mean (SEM).

\section{Supplementary Material}

Refer to Web version on PubMed Central for supplementary material.

\section{Acknowledgments}

We thank Michael Rosenblatt for supplying us with the luciferase-expressing MDA-MB-231 breast cancer cell line. This work was supported by NIH grant P41 EB002520-05 (Tissue Engineering Resource Center) (D.L.K.). F.P.S. is supported by a Mildred Scheel Postdoctoral fellowship from the German Cancer Aid.

\section{References}

1. American Cancer Society: Cancer Facts and Figures 2011. Last accessed December 1, 2011. http:// www.cancer.org/acs/groups/content/@epidemiologysurveilance/documents/document/ acspc-029771.pdf.

2. Bland, K.; Copeland, E. The breast. Comprehensive mangement of benign and malignant disorders. Philadelphia: Saunders; 2004.

3. Fisher B, Anderson S, Bryant J, Margolese RG, Deutsch M, Fisher ER, Jeong JH, Wolmark N. N. Engl. J. Med. 2002; 347:1233. [PubMed: 12393820]

4. Veronesi U, Cascinelli N, Mariani L, Greco M, Saccozzi R, Luini A, Aguilar M, Marubini E. N. Engl. J. Med. 2002; 347:1227. [PubMed: 12393819]

5. Overgaard M, Hansen PS, Overgaard J, Rose C, Andersson M, Bach F, Kjaer M, Gadeberg CC, Mouridsen HT, Jensen MB, Zedeler K. N. Engl. J. Med. 1997; 337:949. [PubMed: 9395428]

6. Brem H, Piantadosi S, Burger PC, Walker M, Selker R, Vick NA, Black K, Sisti M, Brem S, Mohr G, Muller P, Morawetz R, Schold SC. Lancet. 1995; 345:1008. [PubMed: 7723496]

7. Westphal M, Ram Z, Riddle V, Hilt D, Bortey E. Acta Neurochir. 2006; 148:269. [PubMed: 16482400]

8. Al-Abd AM, Hong KY, Song SC, Kuh HJ. J. Control. Release. 2010; 142:101. [PubMed: 19819274]

9. Dadsetan M, Liu Z, Pumberger M, Giraldo CV, Ruesink T, Lu L, Yaszemski MJ. Biomaterials. 2010; 31:8051. [PubMed: 20696470]

10. Chang G, Ci T, Yu L, Ding J. J. Control. Release. 2011; 156:21. [PubMed: 21777632]

11. Zhao L, Zhu L, Liu F, Liu C, Shan D, Wang Q, Zhang C, Li J, Liu J, Qu X, Yang Z. Int. J. Pharm. 2011; 410:83. [PubMed: 21421032]

12. Guo DD, Hong SH, Jiang HL, Kim JH, Minai-Tehrani A, Kim JE, Shin JY, Jiang T, Kim YK, Choi YJ, Cho CS, Cho MH. Biomaterials. 2012; 33:2272. [PubMed: 22177840]

13. Wolinsky JB, Liu R, Walpole J, Chirieac LR, Colson YL, Grinstaff MW. J. Control. Release. 2010; 144:280. [PubMed: 20184934]

14. Ulbrich K, Strohalm J, Kopecek J. Biomaterials. 1982; 3:150. [PubMed: 7115858]

15. Omenetto FG, Kaplan DL. Science. 2010; 329:528. [PubMed: 20671180]

16. Vepari C, Kaplan DL. Prog. Polym. Sci. 2007; 32:991. [PubMed: 19543442]

17. Pritchard EM, Kaplan DL. Expert. Opin. Drug Deliv. 2011; 8:797. [PubMed: 21453189]

18. Wenk E, Merkle HP, Meinel L. J. Control. Release. 2011; 150:128. [PubMed: 21059377]

19. Hines DJ, Kaplan DL. Biomacromolecules. 2011; 12:804. [PubMed: 21250666] 
20. Weiss RB. Semin. Oncol. 1992; 19:670. [PubMed: 1462166]

21. Shan K, Lincoff AM, Young JB. Ann. Intern. Med. 1996; 125:47. [PubMed: 8644988]

22. Doroshow JH, Locker GY, Ifrim I, Myers CE. J. Clin. Invest. 1981; 68:1053. [PubMed: 7287901]

23. Gupta GP, Massague J. Cell. 2006; 127:679. [PubMed: 17110329]

24. Tobias, J.; Hochhauser, D. Cancer and its management. 6ed. Oxford, UK: Wiley-Blackwell; 2010.

25. Wang X, Kluge JA, Leisk GG, Kaplan DL. Biomaterials. 2008; 29:1054. [PubMed: 18031805]

26. Jiang B, Zhang L, Wang Y, Li M, Wu W, Guan S, Liu X, Yang M, Wang J, Guo DA. Food Chem. Toxicol. 2009; 47:1538. [PubMed: 19358873]

27. Zhu J, Zhang J, Zhang L, Du R, Xiang D, Wu M, Zhang R, Han W. Biomed. Pharmacother. 2011; 65:481. [PubMed: 22000485]

28. Yucel T, Cebe P, Kaplan DL. Biophys. J. 2009; 97:2044. [PubMed: 19804736]

29. Rockwood DN, Preda RC, Yucel T, Wang X, Lovett ML, Kaplan DL. Nat. Protoc. 2011; 6:1612. [PubMed: 21959241]

30. Goldstein RH, Reagan MR, Anderson K, Kaplan DL, Rosenblatt M. Cancer Res. 2010; 70:10044. [PubMed: 21159629]

31. Bertinchant JP, Polge A, Juan JM, Oliva-Lauraire MC, Giuliani I, Marty-Double C, Burdy JY, Fabbro-Peray P, Laprade M, Bali JP, Granier C, de la Coussaye JE, Dauzat M. Clin. Chim. Acta. 2003; 329:39. [PubMed: 12589964] 
A

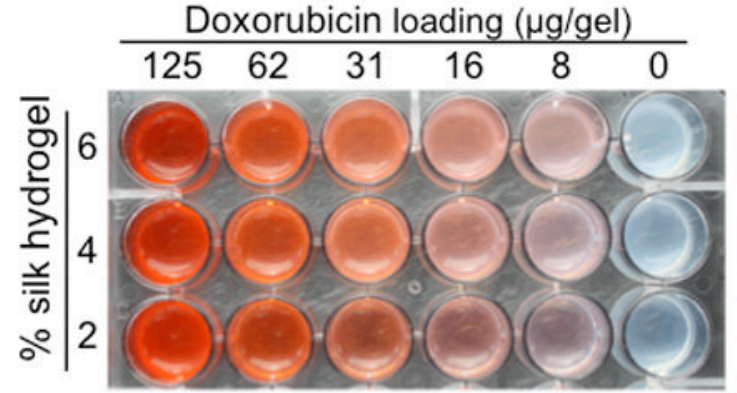

B

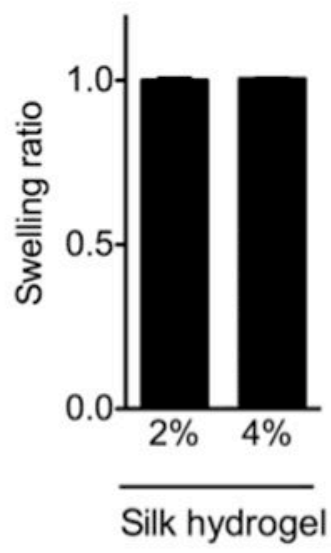

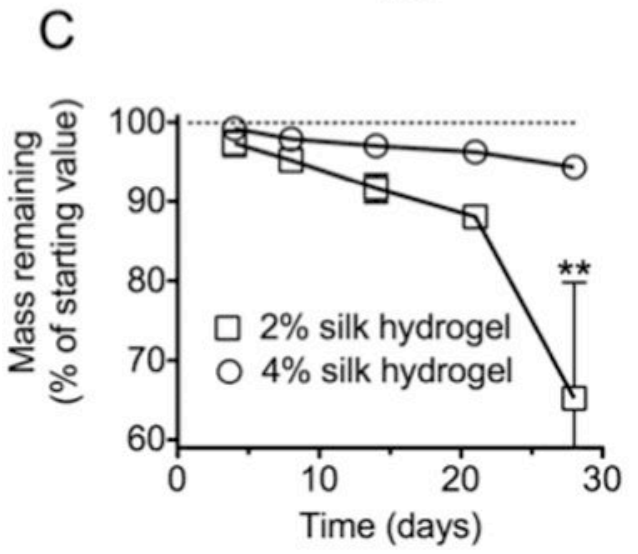

E Doxorubicin loading $(\mu \mathrm{g} / \mathrm{gel})$

Figure 1.

Formation and properties of doxorubicin-loaded silk hydrogels. (A) Impact of silk content and processing time on gel formation. (B) Swelling of silk hydrogels after incubation in PBS for 24 h. (C) Degradation of silk hydrogels in PBS (dotted line) and a-chymotrypsin over 4 weeks. (Statistical differences were determined with Student's t-test, ${ }^{*} * \mathrm{P}<0.001 ; \pm \mathrm{SD}$; $\mathrm{n}=4$ ). (D) Impact of silk content and degumming time (i.e., boiling time during silk purification) on the cumulative doxorubicin release into PBS. (Statistical differences between $6 \mathrm{wt} \%$ silk hydrogels and other groups were determined with ANOVA followed by Dunnett's multiple comparison post hoc test, $* * \mathrm{P}<0.001$, $* * * \mathrm{P}<0.0001 ; \pm \mathrm{SD} ; \mathrm{n}=4)$. (E) 
Images of silk hydrogels with varied amounts of silk and doxorubicin. (F) Injectability of a $2 \mathrm{wt} \%$ doxorubicin silk hydrogel from a pre-filled syringe. 
A

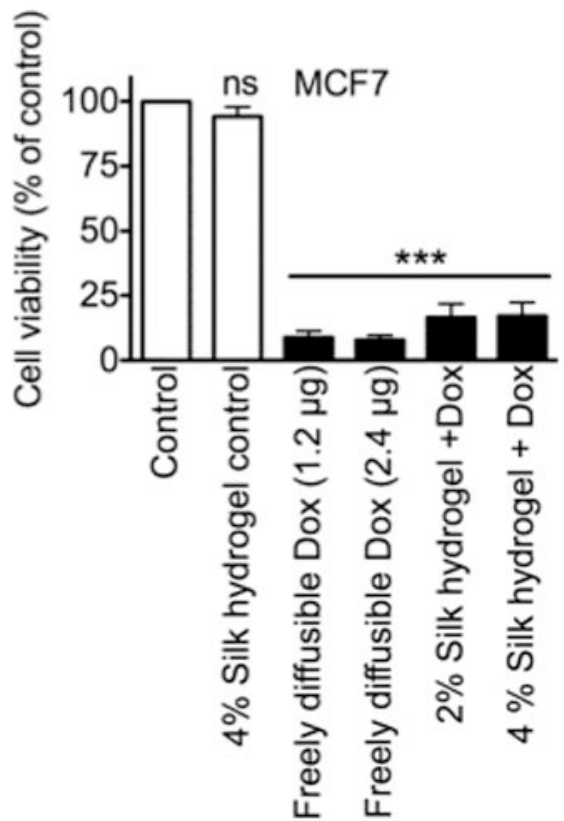

$\mathrm{B}$

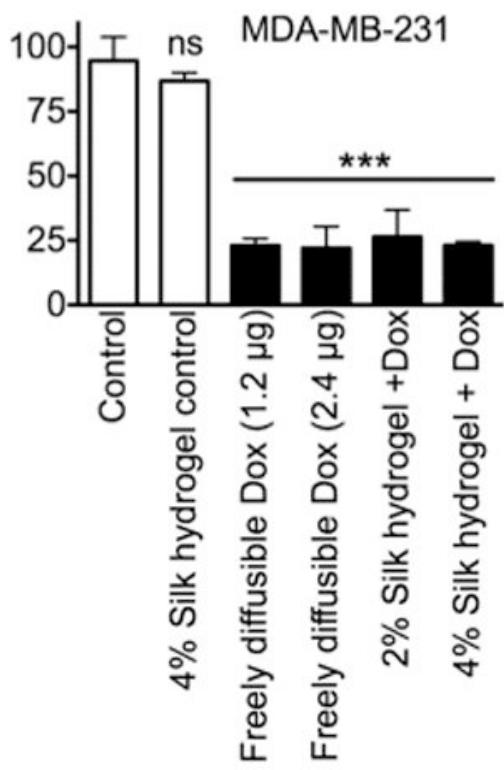

\section{C $\nabla$ Control}

Doxorubicin + silk hydrogel $\square 2 \%$ or $\bigcirc 4 \%$

Freely diffusible doxorubicin $\times 1.2 \mu \mathrm{g}, \diamond 2.4 \mu \mathrm{g}$ or $\diamond 40 \mu \mathrm{g}$
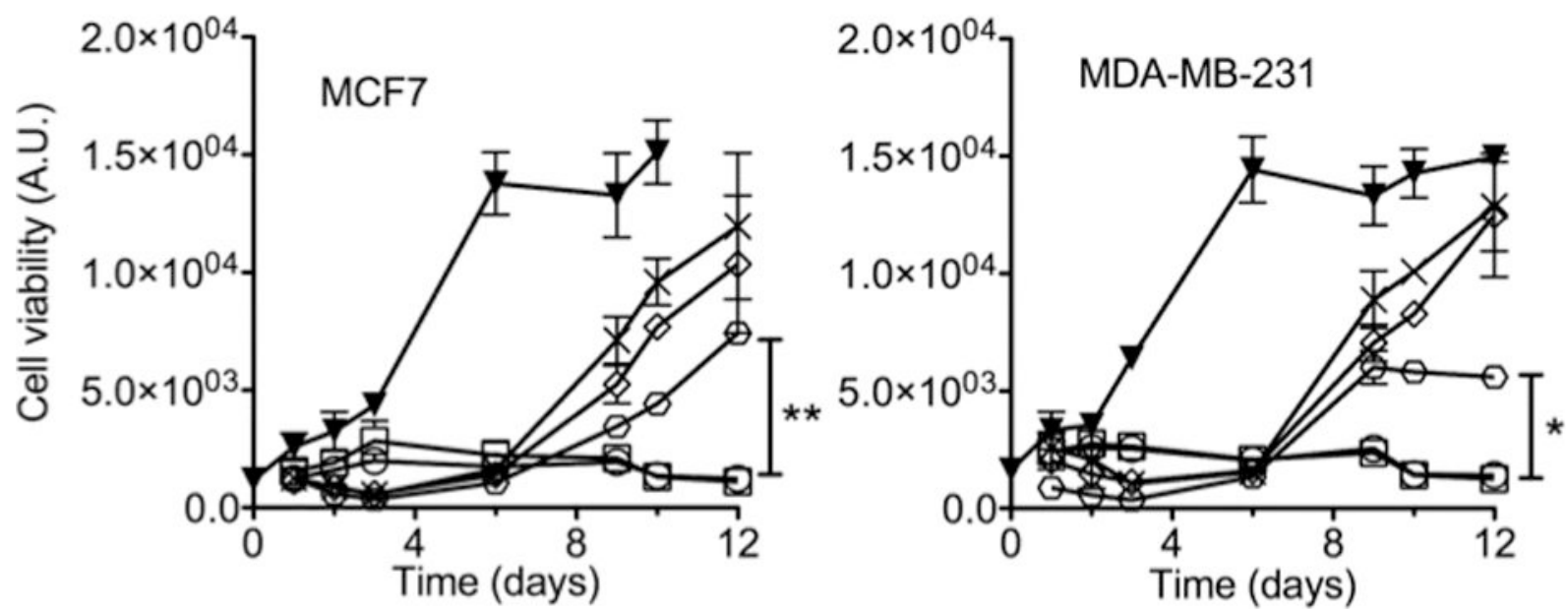

Figure 2.

Improved in vitro toxicity of doxorubicin-loaded silk hydrogels towards human breast cancer cells. (A) MCF-7 and (B) MDA-MB-231 cell viability following a $72 \mathrm{~h}$ exposure to doxorubicin-loaded silk hydrogels ( $40 \mu \mathrm{g}$ of doxorubicin/gel), or free doxorubicin that corresponds to the amount of doxorubicin released from gels during the incubation period. (Statistical differences between the control and other groups was determined with ANOVA followed by Dunnett's multiple comparison post hoc test, ns=not significant, ${ }^{*} * * \mathrm{P}<0.0001$; $\pm \mathrm{SD}$; $=3$ ). (C) Long term toxicity of free doxorubicin and silk hydrogels loaded with doxorubicin. Culture medium was replaced at the indicated time. With the exception of 
control wells, wells were re-seeded with the corresponding breast cancer cells at day 6 . (Statistical differences were determined with ANOVA followed by Bonferroni's multiple comparison post hoc test, ${ }^{*} \mathrm{P}<0.05$, $* * \mathrm{P}<0.001 ; \pm \mathrm{SD} ; \mathrm{n}=4$ ). 

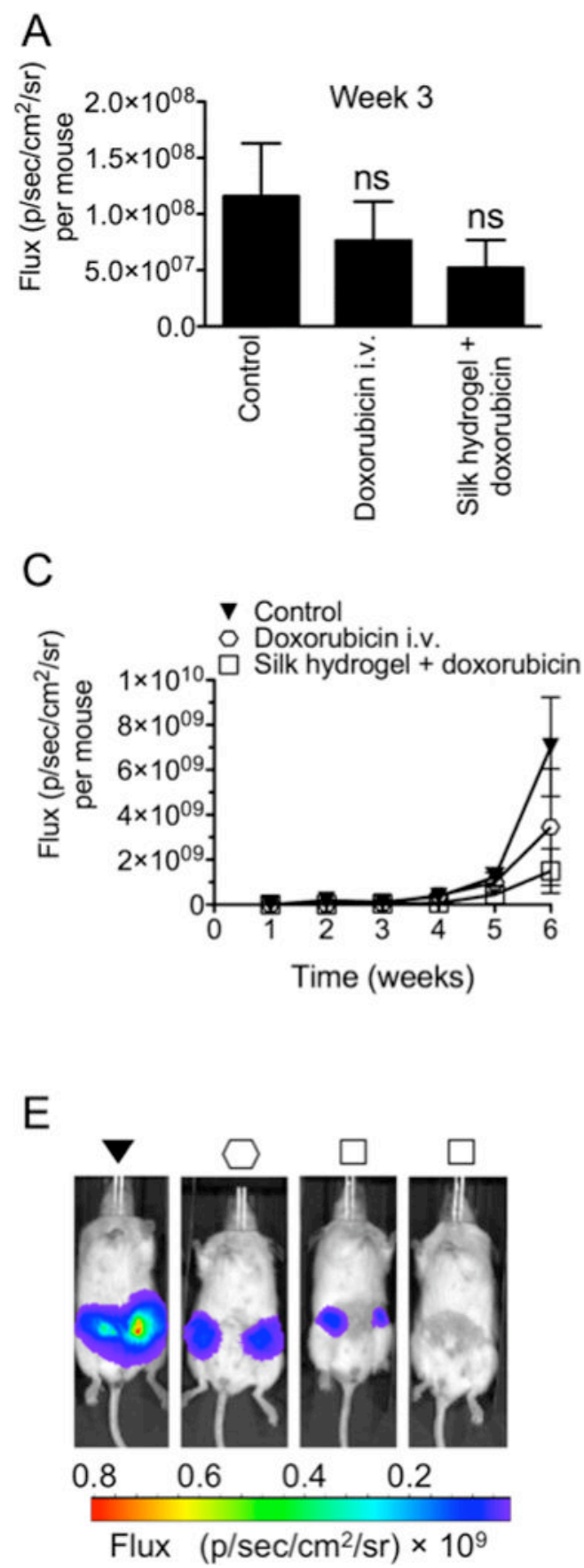

B

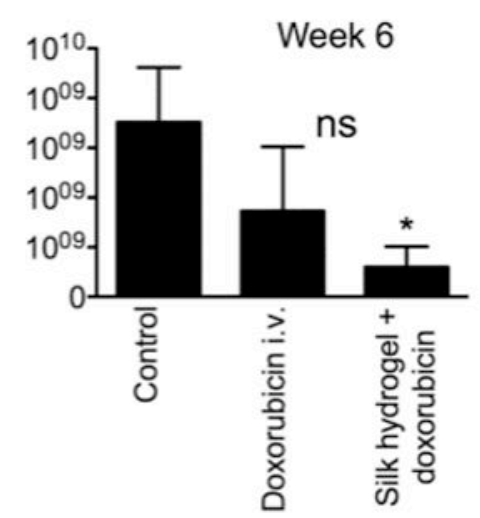

D

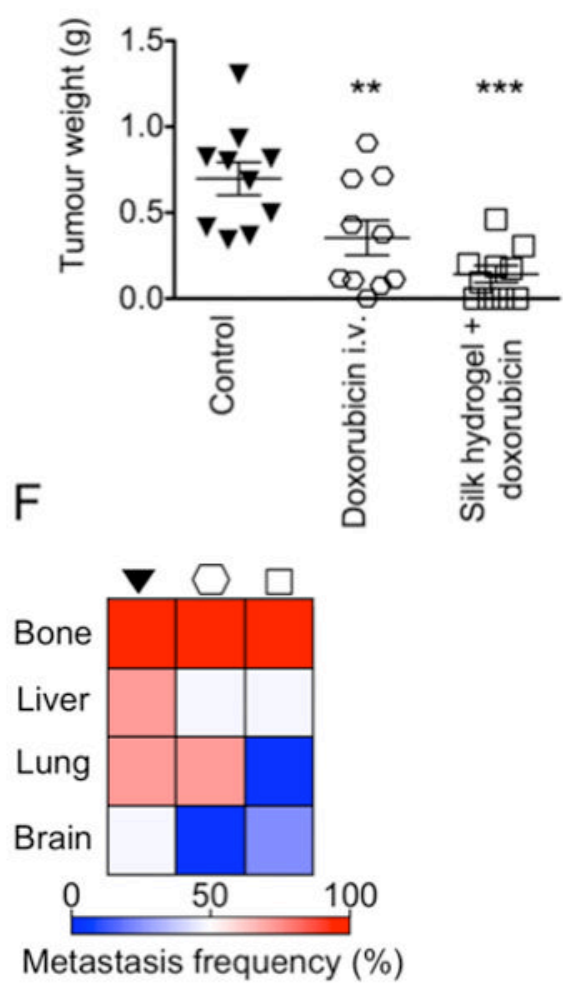

Figure 3.

In vivo response of orthotopic breast tumors to doxorubicin-loaded silk hydrogels. (A) Cancer cell-associated bioluminescence at week 3, (B) 6 and (C) over the time course of the study. (D) Weight of primary tumors at the end of the study (week 6). (E) In vivo tumor cell specific bioluminescence of representative mice from each treatment group at week 6. Plot symbols are defined in panel (C). Two mice treated with doxorubicin-containing silk hydrogels showed complete tumor regression. (F) Metastatic spread of cancer cells to organs at week 6. (Plot symbols are defined in panel (C). (ns=not significant, $* \mathrm{P}<0.005$, $* * \mathrm{P}<0.001$; \pm SEM; $n=5$ ). 
A

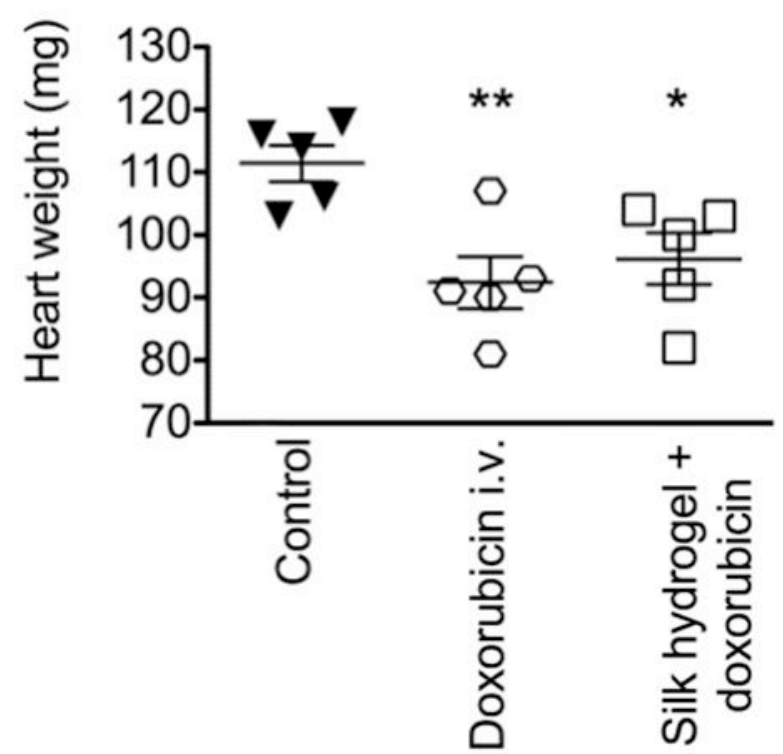

B

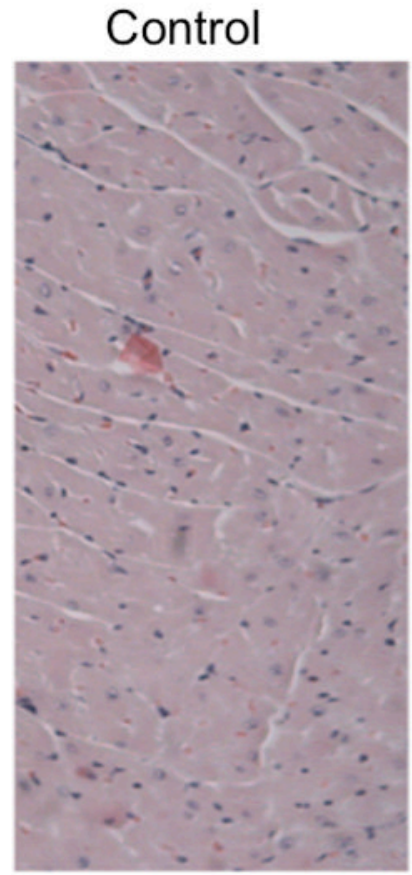

Silk hydrogel +

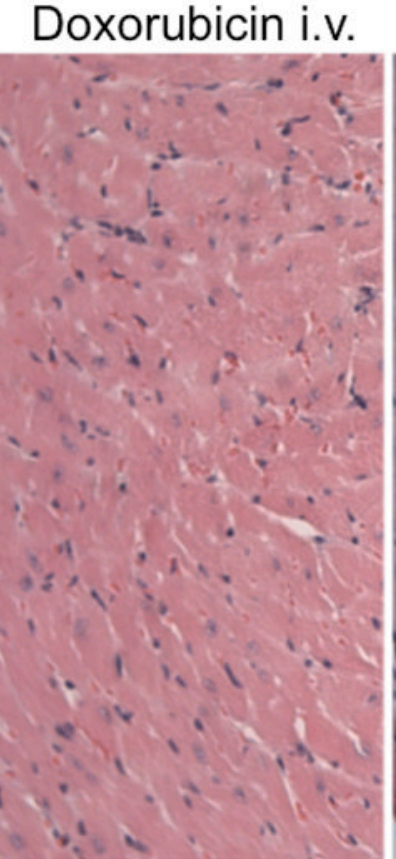

doxorubicin

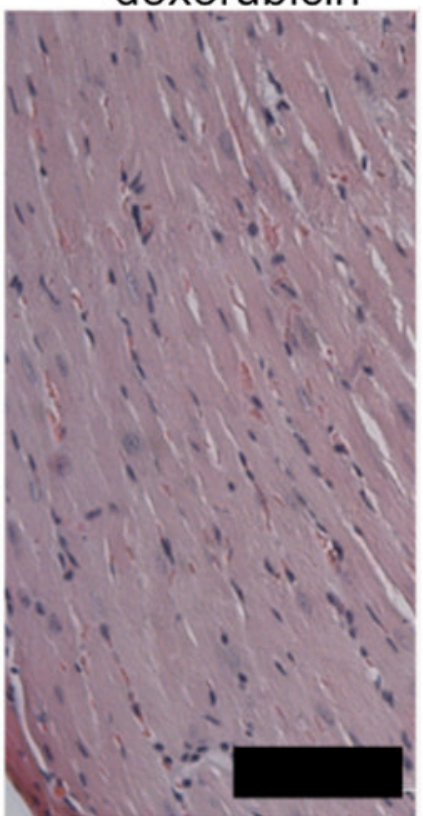

Figure 4.

In vivo toxicity following systemic and local doxorubicin application. (A) Heart weight of animals at week 6. (Statistical differences between control animals and the other treatment groups was determined with ANOVA followed by Dunnett's multiple comparison post hoc test, ns=not significant, ${ }^{*} \mathrm{P}<0.001,{ }^{*} * * \mathrm{P}<0.0001 ; \pm \mathrm{SEM} ; \mathrm{n}=5$ ). (B) Histological section and H\&E staining of hearts at week 6. (Scale bar is $100 \mu \mathrm{m}$ ). 